Quim. Nova, Vol. 35, No. 9, 1794-1799, 2012

\title{
CARACTERIZAÇÃO E CLASSIFICAÇÃO QUANTO AO RISCO AMBIENTAL DO ESTÉRIL DA MINA DE CROMITA DO MUNICÍPIO DE ANDORINHA, BAHIA
}

\author{
Aline Maria dos Santos Teixeira*, Francisco M. S. Garrido e Marta Eloisa Medeiros \\ Departamento de Química Inorgânica, Instituto de Química, Universidade Federal do Rio de Janeiro, Av. Athos da Silveira Ramos, \\ 149, B1. A, 21941-909 Rio de Janeiro - RJ, Brasil \\ João Alves Sampaio \\ Centro de Tecnologia Mineral, Ministério da Ciência, Tecnologia e Inovação, Av. Pedro Calmon, 900, 21941-908 Rio de Janeiro \\ - RJ, Brasil \\ Recebido em 10/2/12; aceito em 11/6/12; publicado na web em 14/8/12
}

\begin{abstract}
CHARACTERIZATION AND CLASSIFICATION OF THE ENVIRONMENTAL RISK OF HOST ROCK FROM A CHROMITE MINE IN ANDORINHA / BAHIA / BRAZIL. The mineral waste studied was host rock from a chromite mine located in Andorinha/Bahia, extracted and stockpiled in yards without specific application. Host rock was submitted to chemical analysis, XRD, SEM-EDS, IR and TGA and classified according to ABNT standards for solid waste classification. Analyses confirmed that this host rock, classified as ultrabasic, consists mainly of dolomite, calcite and diopside. Hazard assessment results showed this host rock should be classified as class II B - inert waste, important for its potential application in agriculture as a soil acidity correction agent.
\end{abstract}

Keywords: mineral waste; soil acidity corrective; hazardous materials.

\section{INTRODUÇÃO}

A identificação de fontes alternativas para a agricultura originou novos grupos de insumos agrícolas. A utilização, principalmente, de lodos provenientes de estações de tratamento de água, escórias de siderurgias e rejeitos de lixiviação química do caulim, na agricultura, tem convertido o destino destes rejeitos, de modo a diminuir o impacto ambiental em torno destas indústrias. Assim, a definição de métodos de processamento e a avaliação sistemática destas novas fontes alternativas, para a correção e fertilização do solo, tornaram-se importantes para os atuais modelos de produção agrícola. ${ }^{1-4}$

O Ministério da Agricultura, Pecuária e Abastecimento (MAPA), por meio da Instrução Normativa de 4/7/2006, estabelece sobre a definição, especificação, métodos analíticos, comercialização de corretivos de acidez, de alcalinidade e sodicidade, e dos condicionadores de solo destinados à agricultura. Esta Instrução Normativa define, como corretivo de acidez, o produto que promove a correção da acidez do solo, além de fornecer cálcio, magnésio ou ambos. ${ }^{5}$

As rochas básicas e ultrabásicas são consideradas ótimas fornecedoras de nutrientes minerais para o solo. ${ }^{1}$ Dentre estas, destacam-se os serpentinitos que são rochas metamórficas ultrabásicas, formadas principalmente pelos óxidos de cálcio, magnésio e silício. Deve-se considerar que estas rochas podem apresentar variações na sua composição mineralógica, podendo ser parcialmente convertidas em carbonato, mas preservando alguns dos compostos mineralógicos originais da rocha. As reservas destas rochas estão bem distribuídas no Brasil e podem ser encontradas nos estados da Bahia, Ceará, Goiás, Minas Gerais, Mato Grosso do Sul, Pará, Paraíba, Paraná, Rio Grande do Sul, Sergipe e São Paulo. ${ }^{6}$

O uso de rochas na agricultura consiste em uma técnica de fertilização natural que, além de corrigir a acidez do solo, contribui com a reposição dos nutrientes, o que reduz o consumo de fertilizantes industriais, visto que grande parte destes fertilizantes é procedente de fontes não renováveis e, muitas vezes, importados. Esta técnica,

*e-mail: haline_santos@ufrj.br denominada de rochagem (rocks for crops), se resume na adição do pó de rocha ao solo que, pelo intemperismo químico, em que a água é o principal solvente, decompõe o pó de rocha lentamente, liberando os nutrientes gradualmente. ${ }^{1}$

Os efeitos benéficos para a fertilidade do solo e a nutrição das plantas podem ampliar o potencial do uso de rochas como corretivos de solos e, desta forma, agregar valor a estas novas fontes de insumos alternativos. Estas rochas são, muitas vezes, provenientes do descarte de jazidas, denominado de estéril de mineração, uma vez que não possuem o minério bruto ou cujo teor de minerais que torna inviável seu tratamento ou concentração mineral. ${ }^{1,4,7}$ Contudo, a utilização destas rochas na agricultura deve ser exaustivamente estudada, assim como avaliado o impacto ambiental que este processo pode acarretar, ${ }^{7}$ principalmente, pelo fato destas novas fontes de insumos possuírem uma composição química variada. Ademais, aplicações em altas quantidades destes materiais podem implicar em um desequilíbrio nutricional ou em um acúmulo de elementos potencialmente tóxicos no solo, de modo que é imprescindível avaliar a viabilidade de aplicação de cada rocha, ${ }^{1,8,9}$ principalmente no caso de agentes mutagênicos e carcinogênicos, como o cromo, que na forma hexavalente é tóxico para animais e humanos.

Embora o cromo exista em diversos estados de oxidação, as formas mais comuns de ocorrência são a trivalente e a hexavalente. A presença de $\mathrm{Cr}$ (III), estado de oxidação mais estável, é importante na dieta de animais e humanos, pois está relacionada com o metabolismo de glicose, lipídeos e proteínas. Contudo, o $\mathrm{Cr}(\mathrm{VI})$ é tóxico e possui maior mobilidade que o $\mathrm{Cr}(\mathrm{III})$. É necessário um monitoramento ambiental adequado, já que o cromo e seus compostos são abundantemente empregados em processos industriais e quantidades significativas de materiais contendo este elemento são descartadas na natureza em sistemas atmosféricos, aquáticos e no solo. ${ }^{10}$

O Conselho Nacional do Meio Ambiente (CONAMA) elaborou uma resolução que define critérios e valores orientadores de qualidade do solo e águas subterrâneas quanto à presença de substâncias químicas. Essa resolução, também, estabelece diretrizes para o monitoramento de áreas contaminadas por essas substâncias em decorrência de atividades antrópicas. ${ }^{11}$ 
A Associação Brasileira de Normas Técnicas (ABNT) classifica os resíduos com base em sua periculosidade e solubilidade, avaliando o seu risco potencial ao meio ambiente e à saúde pública, de forma a indicar o manuseio e o destino apropriados. Deste modo, os resíduos podem ser classificados em: classe I - perigosos e classe II - não perigosos. Esta última classe é subdividida em resíduos classe II A - não inertes e classe II B - inertes. A periculosidade é medida em função das propriedades físicas e químicas ou infectocontagiosas associadas ao material. ${ }^{12}$ Os resíduos classe I - perigosos - são aqueles que possuem características de inflamabilidade, corrosividade, reatividade, toxicidade e patogenicidade. Os resíduos classe II A - não inertes - podem possuir propriedades como combustibilidade, biodegradabilidade e/ou solubilidade em água. Os resíduos da classe II B - inertes - correspondem a aqueles que, quando submetidos a testes de lixiviação e solubilidade, não disponibilizam ou não dispõem de constituintes solubilizados em concentrações superiores aos limites estabelecidos pela Normativa. ${ }^{12}$

A legislação, de modo geral, prevê que a responsabilidade da aplicação de um rejeito seja da fonte geradora e que esta deva garantir a rastreabilidade e monitoramento das áreas que irão receber esse material. Por outro lado, quando o resíduo não atender às especificações determinadas por meio dos órgãos responsáveis pelo controle ambiental, mesmo após tratamentos, deve ser descartado e disposto em local apropriado, de maneira que não seja utilizado. ${ }^{9}$

Nesta visão, o presente trabalho teve como objetivos principais caracterizar, segundo os aspectos químico, estrutural e físico, o estéril da mina de cromita $\left(\mathrm{FeOCr}_{2} \mathrm{O}_{3}\right)$ do município de Andorinha/BA, classificá-lo de acordo com a sua periculosidade, conforme as normas técnicas da ABNT, além de avaliar a questão ambiental do cromo, já que se trata do estéril de uma mina de cromita. A capacidade da mina para obtenção da cromita é em torno de 20.000 t/mês, sendo que a relação minério:estéril é de $1: 4$, com teor médio da cromita de $38 \%$ de $\mathrm{Cr}_{2} \mathrm{O}_{3}$ e $17 \%$ de $\mathrm{FeO}$. Cabe ressaltar que existe cerca de $250.000 \mathrm{t}$ da rocha em estudo, lavrada e acumulada em pátios, sem definição de aplicação, o que gera um impacto ambiental na mina, causado pelo acúmulo deste estéril.

\section{PARTE EXPERIMENTAL}

\section{Pré-preparo das amostras}

O estudo do estéril da mina de cromita, pertencente ao grupo FERBASA, do município de Andorinha/BA, teve início com o recebimento de $200 \mathrm{~kg}$ de amostra da rocha pelo CETEM/MCTI. A rocha foi submetida às etapas de britagem, moagem, homogeneização e quarteamento, para assegurar a representabilidade das amostras. Nesta etapa foram obtidas alíquotas com $1 \mathrm{~kg}$ da rocha com granulometria abaixo de $4,7 \mathrm{~mm} .{ }^{13}$ Uma alíquota de $1 \mathrm{~kg}$ da amostra foi quarteada e uma das frações foi cominuída a granulometria abaixo de $0,147 \mathrm{~mm}$, o que proporcionou a obtenção de amostras com massa, granulometria e homogeneidade adequadas à análise de composição e caracterização mineralógica.

\section{Classificação da rocha quanto à sua periculosidade e solubilidade}

A classificação do estéril quanto à sua periculosidade e solubilidade, com base em seu risco potencial ao meio ambiente e à saúde pública, foi realizada segundo os procedimentos descritos nas normas técnicas da ABNT. ${ }^{12}$ Para tanto, uma alíquota de $1 \mathrm{~kg}$ da amostra foi cominuída a uma granulometria abaixo de $2 \mathrm{~mm}$, conforme especificação granulométrica para corretivo de acidez, estabelecido pelo MAPA. ${ }^{5}$
A avaliação da periculosidade do material foi realizada segundo a norma NBR-10005, que avalia a toxicidade do resíduo e define o limite máximo da concentração dos poluentes no extrato lixiviado. Este ensaio se resume na análise química do extrato lixiviado do material com uma solução de extração, definida pelo valor de $\mathrm{pH}$ observado em uma suspensão contendo $5 \mathrm{~g}$ da amostra e $96,5 \mathrm{~mL}$ de água deionizada. Se o valor de $\mathrm{pH}$ medido for superior a 5,0 deve-se adicionar 3,5 mL de $\mathrm{HCl} 1 \mathrm{~mol} \mathrm{~L}^{-1}$ à solução, aquecer a $50{ }^{\circ} \mathrm{C}$ durante 10 min e determinar o novo valor de $\mathrm{pH}$ da solução depois de resfriada. $\mathrm{O}$ pH medido na suspensão do estéril em meio aquoso foi de 9,70 e o pH obtido após adição do ácido foi de 5,26. Assim, a solução de extração, a ser utilizada no teste de lixiviação corresponde a uma solução de ácido acético $0,1 \mathrm{~mol} \mathrm{~L}^{-1} \mathrm{com}$ pH em torno de 2,88 $\pm 0,05$, conforme estabelecido na Norma Técnica. ${ }^{12}$

No teste de lixiviação uma mistura, que continha o material e a solução extratora na proporção de 1:20, foi agitada a $300 \mathrm{rpm}$ em um shaker, MA-420 Marconi, na temperatura ambiente, por um período de $18 \mathrm{~h}$. A fase líquida da mistura foi separada da fase sólida por meio da filtração com papel de filtro com $0,8 \mu \mathrm{m}$ de porosidade. Uma prova em branco foi feita a fim de verificar a ocorrência de contaminação durante o ensaio. O extrato lixiviado foi analisado quimicamente pelas técnicas de potenciometria, espectrometrias de absorção atômica com chama $\mathrm{C}_{2} \mathrm{H}_{2} / \operatorname{ar}(\mathrm{AA})$ e absorção atômica com chama $\mathrm{C}_{2} \mathrm{H}_{2} / \mathrm{N}_{2} \mathrm{O} / \mathrm{ar}$ (AA$\mathrm{N}_{2} \mathrm{O}$ ), espectrometria de emissão óptica com plasma indutivamente acoplado (ICP-OES) e espectrofotometria de absorbância na região do visível. Foi avaliado de acordo com o anexo F da norma NBR-10004, que define o limite da concentração de poluentes no extrato obtido no teste de lixiviação. ${ }^{12}$ Ademais, por se tratar da avaliação do impacto ambiental do estéril de uma mina de cromo, foram determinados os teores de cromo total e hexavalente nos extratos lixiviados de amostras com granulometria inferior a 2 e $0,147 \mathrm{~mm}$, já que a área superficial da amostra pode interferir no teor de cromo lixiviado.

A avaliação da solubilidade do material foi realizada conforme a NBR-10006, que diferencia os resíduos da classe II (não perigosos) em classe IIA - não inertes e classe IIB - inertes. ${ }^{12}$ Neste ensaio uma mistura da rocha $(<2 \mathrm{~mm})$ e água, na proporção de 1:4, foi mantida em agitação por 5 min em um shaker, MA-420 Marconi, a 300 rpm na temperatura ambiente. Após agitação, a mistura foi mantida em repouso por 7 dias. $\mathrm{O}$ extrato solubilizado da rocha foi obtido pela filtração da mistura, na qual foi utilizada uma membrana filtrante com $0,45 \mu \mathrm{m}$ de porosidade. As concentrações dos poluentes no extrato solubilizado da rocha foram determinadas quimicamente pelas técnicas de potenciometria, absorção atômica com chama $\mathrm{C}_{2} \mathrm{H}_{2} / \mathrm{ar}$ (AA), cromatografia iônica (CI), espectrometria de emissão óptica com plasma indutivamente acoplado (ICP-OES) e espectrofotometria de absorbância na região do visível e avaliadas segundo os limites definidos no anexo $\mathrm{G}$ da norma NBR-10004. ${ }^{12}$ Os teores de cromo total e hexavalente também foram determinados nos extratos solubilizados das amostras com granulometria inferior a 2 e $0,147 \mathrm{~mm}$.

\section{Abertura de amostras para análise de composição}

Uma amostra da rocha, com granulometria abaixo de $0,147 \mathrm{~mm}$, foi solubilizada em $\mathrm{HCl}$ e o resíduo da solubilização encaminhado para a determinação de sílica $\left(\mathrm{SiO}_{2}\right)$. Para a determinação dos demais cátions, a rocha foi digerida pela adição de $\mathrm{HCl}, \mathrm{HNO}_{3}$ e $\mathrm{HF}$; os sais formados foram solubilizados em $\mathrm{HNO}_{3}$ e a solução foi encaminhada para análise.

\section{Descrição das análises}

As análises químicas foram realizadas nos laboratórios da Coordenação de Análises Minerais (COAM/CETEM). Foram 
utilizadas as técnicas de gravimetria, cromatografia iônica (CI), absorção atômica com chama $\mathrm{C}_{2} \mathrm{H}_{2} / \mathrm{ar}$ (AA), absorção atômica com chama $\mathrm{C}_{2} \mathrm{H}_{2} / \mathrm{N}_{2} \mathrm{O} / \operatorname{ar}\left(\mathrm{AA}-\mathrm{N}_{2} \mathrm{O}\right)$, espectrometria de emissão óptica com plasma indutivamente acoplado (ICP-OES) e espectrofotometria de absorbância na região do visível. Todas as soluções analíticas foram preparadas a partir de reagentes de grau analítico e água ultrapura obtida com um sistema de purificação de água Milli-Q.

$\mathrm{O}$ teor de $\mathrm{SiO}_{2}$ foi analisado por gravimetria utilizando $\mathrm{HF}$ e $\mathrm{H}_{2} \mathrm{SO}_{4}$. Os íons fluoreto e cianeto foram analisados por potenciometria. As determinações por ICP-OES foram realizadas em um equipamento Horiba Jobin Yvon, modelo Ultima 2, com vista de observação radial do plasma, os elementos estudados e as linhas espectrais foram: $\mathrm{Al}(396,152 \mathrm{~nm}), \mathrm{Fe}(259,940 \mathrm{~nm}), \mathrm{Ca}(422,673$ $\mathrm{nm}), \mathrm{Mg}(280,270 \mathrm{~nm}), \mathrm{P}(214,914 \mathrm{~nm}), \mathrm{Ni}(231,604 \mathrm{~nm}), \mathrm{Mn}$ (259,373 nm), As (197,198 nm), Pb (220,353 nm), Hg (194,164 nm), Ag $(338,289 \mathrm{~nm}), \mathrm{Se}(196,090 \mathrm{~nm}), \mathrm{Cr}(267,716 \mathrm{~nm}), \mathrm{Ba}(455,403$ $\mathrm{nm})$ e Cd (228,802 nm).

As análises por absorção atômica foram realizadas em um espectrômetro Varian, modelo SpectrAA-55B, com lâmpada de catodo oco dos respectivos elementos e os seguintes comprimentos de onda: $\mathrm{Ba}$ (553,6 nm), Cr (357,9 nm), Cd (228,8 nm), Cu (324,8 nm), Fe (248,3 $\mathrm{nm}), \mathrm{Hg}(253,7 \mathrm{~nm}), \mathrm{Na}(589,0 \mathrm{~nm}), \mathrm{Zn}(213,9 \mathrm{~nm}), \mathrm{K}(766,5 \mathrm{~nm}) \mathrm{e}$ $\mathrm{Pb}(217,0 \mathrm{~nm})$; fendas de 0,2 nm (Cr, Fe), 0,5 nm (Ba, Cd, Cu, Hg, $\mathrm{Na})$ e 1,0 nm (Zn, K, Pb); com chama $\mathrm{C}_{2} \mathrm{H}_{2} / \mathrm{ar}(\mathrm{Cd}, \mathrm{Cu}, \mathrm{Fe}, \mathrm{Hg}, \mathrm{Na}$, $\mathrm{Zn}, \mathrm{K}, \mathrm{Pb}$ ) e chama $\mathrm{C}_{2} \mathrm{H}_{2} / \mathrm{N}_{2} \mathrm{O} / \mathrm{ar}(\mathrm{Ba}, \mathrm{Cr})$.

Os teores de cromo hexavalente foram determinados em um espectrofotômetro de absorbância da Perkin Elmer, modelo Lambda 12 , com celas de quartzo, reagente difenilcarbazida em meio de $\mathrm{H}_{2} \mathrm{SO}_{4}$ $0,1 \mathrm{~mol} \mathrm{~L}^{-1}(\mathrm{pH}=1,0)$ e comprimento de onda de $540 \mathrm{~nm}$. Os ânions cloreto, fluoreto, nitrato e sulfato foram determinados em cromatógrafo de íons Metrohm, com um amostrador automático acoplado a um sistema de diálise modelo 788 IC, um degasificador modelo 837 IC e um detector condutimétrico modelo 819 IC, usando uma coluna Metrosep A Supp 10250/4,0 e com solução de $\mathrm{H}_{2} \mathrm{SO}_{4}$ 0,1 mol $\mathrm{L}^{-1}$ utilizada como supressor químico. A perda de massa do estéril após calcinação à $1200^{\circ} \mathrm{C}$, denominada de perda ao fogo, conforme a nomenclatura utilizada na análise de rochas, foi realizada por meio da análise gravimétrica com calcinação à $1200^{\circ} \mathrm{C}$, até peso constante.

\section{Caracterização mineralógica}

Para tal, foram utilizadas as técnicas de difração de raios $\mathrm{X}$ (DRX), microscopia eletrônica de varredura acoplada a espectrômetro dispersivo de energia (MEV/EDS), espectroscopia no infravermelho (IV) e análise termogravimétrica (ATG).

A DRX foi realizada, no equipamento Bruker-D4 Endeavor, com passo do goniômetro de $0,02^{\circ}$ em $2 \theta$, com 1,0 s de tempo de contagem e radiação Co-k $\alpha(\lambda=1,789 \AA ; 35 \mathrm{kV} / 40 \mathrm{~mA})$, na faixa angular $(2 \theta)$ variando de $5^{\circ}$ a $80^{\circ}$. A interpretação qualitativa do difratograma foi efetuada por comparação com os padrões contidos no banco de dados PDF02 em software Bruker EVA. ${ }^{14}$

As medidas de MEV-EDS foram feitas no aparelho LEO S440, em modo de alto vácuo, no qual as partículas foram acondicionadas em suporte próprio e recobertas com prata $(\mathrm{Ag})$, pelo método arco voltaico e injeção por vácuo, formando uma camada de cerca de 20 $\mathrm{nm}$ de prata. $\mathrm{O}$ espectro no infravermelho do estéril foi obtido por meio de uma pastilha de $\mathrm{KBr}$ anidro. Esta análise foi realizada no equipamento Magna 760, marca Nicolet, na região de 4000 a 400 $\mathrm{cm}^{-1}$ e resolução de $4 \mathrm{~cm}^{-1}$.

Para a análise termogravimétrica (ATG) foi utilizado o equipamento TA Instruments, modelo 2690 STD V 3,0 F, na faixa de temperatura de $25^{\circ} \mathrm{C}$ até $1.200^{\circ} \mathrm{C}$, com velocidade de aquecimento de $10{ }^{\circ} \mathrm{C} \mathrm{min}^{-1}$, sob atmosfera oxidante, com fluxo de $110 \mathrm{~mL} \mathrm{~min}^{-1}$ de ar sintético seco filtrado. A massa da amostra analisada foi de aproximadamente $40 \mathrm{mg}$, em cadinho de alumina.

\section{RESULTADOS E DISCUSSÃO}

A composição química $(\% \mathrm{~m} / \mathrm{m})$ do estéril está relacionada na Tabela 1 , assim como a perda ao fogo à $1200^{\circ} \mathrm{C}$. Os resultados da composição química foram expressos em base calcinada, considerando a forma de óxidos binários mais estáveis, e normalizados a 100\%, com o intuito de fornecer uma referência dos elementos presentes no material. ${ }^{7,13}$

Tabela 1. Composição química do estéril

\begin{tabular}{|c|c|c|c|}
\hline Composição & $\%$ em massa & $\begin{array}{l}\text { Limite de detec- } \\
\text { ção }\left(\mathrm{mg} \mathrm{L}^{-1}\right)\end{array}$ & Técnica analítica \\
\hline $\mathrm{CaO}$ & 33,44 & 0,06 & ICP-OES \\
\hline $\mathrm{MgO}$ & 20,77 & 0,003 & ICP-OES \\
\hline $\mathrm{SiO}_{2}$ & 13,20 & 1.000 & gravimetria \\
\hline $\mathrm{Na}_{2} \mathrm{O}$ & 0,453 & 0,1 & AA \\
\hline $\mathrm{Fe}_{2} \mathrm{O}_{3}$ & 0,443 & 0,001 & ICP-OES \\
\hline $\mathrm{Al}_{2} \mathrm{O}_{3}$ & 0,404 & 0,2 & ICP-OES \\
\hline $\mathrm{K}_{2} \mathrm{O}$ & 0,221 & 0,1 & AA \\
\hline $\mathrm{MnO}$ & 0,125 & 0,009 & ICP-OES \\
\hline $\mathrm{Cl}^{-}$ & 0,071 & 0,01 & CI \\
\hline $\mathrm{P}_{2} \mathrm{O}_{5}$ & 0,023 & 0,02 & ICP-OES \\
\hline \multirow[t]{2}{*}{$\begin{array}{c}\text { Perda ao fogo } \\
\left(1200^{\circ} \mathrm{C}\right)\end{array}$} & 30,85 & - & gravimetria \\
\hline & ----------- & -----mg/kg----- & - \\
\hline Níquel & 15,4 & 0,1 & AA \\
\hline Cádmio & 0,72 & 0,02 & AA \\
\hline Chumbo & 17,5 & 0,3 & AA \\
\hline Cromo & 21,3 & 0,3 & AA- $\mathrm{N}_{2} \mathrm{O}$ \\
\hline $\begin{array}{c}\text { Cromo } \\
\text { hexavalente }\end{array}$ & $<20$ & 20 & Visível \\
\hline
\end{tabular}

NOTA: Os teores em \% de massa dos elementos, expressos na forma de seus óxidos, foram calculados por meio do fator de conversão estequiométrico usualmente utilizado na análise de rochas. ${ }^{7,13}$

A análise destes resultados indica que o estéril é rico em cálcio e magnésio. Uma avaliação preliminar indicou que o estéril apresenta as especificações estabelecidas pelo MAPA para corretivos de solo., ${ }^{4,5}$ Em testes utilizando a rocha in natura, foram observadas melhorias nas características de um latossolo amarelo inicialmente ácido, tanto em função do seu pH quanto em relação aos seus teores de cálcio, magnésio e alumínio trocáveis. ${ }^{4}$

Com base na classificação química para rochas ígneas, é possível afirmar que o estéril em estudo é uma rocha ultrabásica, pois o teor de sílica $\left(\mathrm{SiO}_{2}\right)$ na sua composição é inferior a $45 \%$. Esta classificação é dada de acordo com o teor de $\mathrm{SiO}_{2}$ na rocha, de modo que a rocha é considerada ácida com teores superiores a 66\%; de 66 a $52 \%$, intermediária; de 52 a $45 \%$, básica e, para teores inferiores a $45 \%$, é classificada como ultrabásica. ${ }^{15}$ Ademais, os teores obtidos de níquel, cádmio, chumbo e cromo são inferiores aos valores de referência para solos destinados à agricultura, 70; 3; 180 e $150 \mathrm{mg} \mathrm{kg}^{-1}$, respectivamente, de acordo com os limites definidos pela resolução do CONAMA. ${ }^{11}$ Ainda, foi verificado que os teores de cádmio e chumbo, obtidos na composição química da rocha, são inferiores aos limites definidos pelo MAPA, 20 e $1000 \mathrm{mg} \mathrm{kg}^{-1}$ respectivamente, 
sendo que a instrução normativa para corretivos de acidez somente define o limite para estes dois elementos. Além disso, os teores de cromo total e hexavalente são inferiores ao limite especificado para fertilizantes minerais, $200 \mathrm{mg} \mathrm{kg}^{-1}$, conforme o MAPA. ${ }^{5}$

No entanto, ao comparar os elementos potencialmente tóxicos contidos no estéril com os teores obtidos em calcários comercializados atualmente, observou-se que a rocha em estudo possui valores inferiores para níquel, cádmio e chumbo, mas teor de cromo total superior aos valores verificados para esses calcários. ${ }^{16}$ Por fim, cabe ressaltar que o teor de cromo total do estéril é menor ou próximo ao teor de cromo de vários solos utilizados para agricultura no Brasil, o que indica que, em princípio, o estéril poderia ser aplicado como corretivo de acidez, entretanto, não seria recomendado seu uso em solos com baixo teor de cromo e/ou com ambientes químicos que possam promover a oxidação do $\mathrm{Cr}(\mathrm{III})$ a $\mathrm{Cr}(\mathrm{VI}){ }^{10,17}$

A análise do difratograma de raios $\mathrm{X}$ do estéril, Figura 1, indica que a composição mineralógica básica é constituída principalmente por dolomita $\left[\mathrm{CaMg}\left(\mathrm{CO}_{3}\right)_{2}\right]$, calcita $\left(\mathrm{CaCO}_{3}\right)$, diopsídio $\left(\mathrm{CaMgSi}_{2} \mathrm{O}_{6}\right)$, muscovita $\left[\mathrm{KAl}_{2} \mathrm{Si}_{3} \mathrm{AlO}_{10}(\mathrm{OH}, \mathrm{F})_{2}\right]$ e antigorita $\left[(\mathrm{Mg}, \mathrm{Fe})_{3} \mathrm{Si}_{2} \mathrm{O}_{5}(\mathrm{OH})_{4}\right]{ }^{4}$ A identificação dos picos relacionados à antigorita sugere que a rocha em estudo pertence ao grupo dos serpentinitos. Ademais, a análise dos resultados de DRX sugere que a rocha tenha sofrido alterações na sua composição mineralógica original, uma vez que os picos mais intensos no difratograma de raios $\mathrm{X}$ do estéril são referentes à dolomita e a calcita. Assim, a rocha em estudo pode ser considerada um mármore serpentina, pois possui uma composição parcialmente convertida em carbonatos, indicando que provavelmente a rocha original sofreu metamorfismo com altas pressões parciais de $\mathrm{CO}_{2}{ }^{6}$

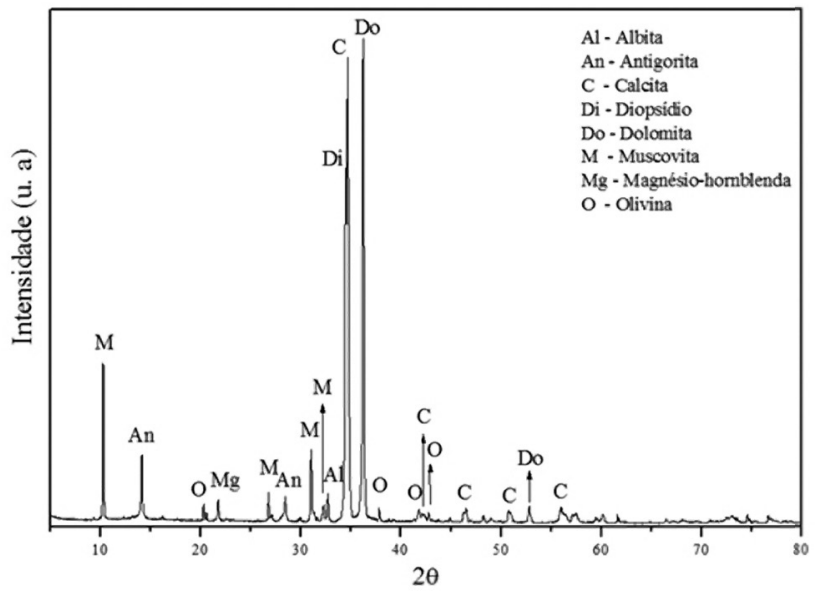

Figura 1. Difratograma de raios $X$ do estéril

A técnica de MEV/EDS, Figura 2, permitiu identificar que a amostra homogeneizada é formada por partículas de morfologias distintas, com forma irregular e dimensões variando de 30 a $200 \mu \mathrm{m}$. Os principais constituintes minerais foram identificados por EDS, corroborando os resultados de DRX.

A Figura 3 ilustra o espectro no infravermelho obtido para uma amostra do estéril. A análise do espectro permitiu atribuir, por tentativa, bandas referentes ao íon carbonato para a calcita (2990, 2880, 2520 e $1800 \mathrm{~cm}^{-1}$ ), assim como outras bandas características das vibrações do íon carbonato (1430, 1090, 876 e $\left.714 \mathrm{~cm}^{-1}\right)$. Foram observadas bandas correspondentes ao estiramento da ligação $\mathrm{O}-\mathrm{H}$ em argilo minerais (3480 e $3410 \mathrm{~cm}^{-1}$ ) e para $\mathrm{Mg}-\mathrm{OH}\left(3690 \mathrm{~cm}^{-1}\right)$ e, ainda, uma banda referente ao íon $\left[\mathrm{SiO}_{4}\right]^{4-}\left(957 \mathrm{~cm}^{-1}\right){ }^{18,19}$

A análise do gráfico com a curva termogravimétrica (ATG) e a derivada (DTG), Figura 4, indica que a temperatura de decomposição da rocha é aproximadamente $800^{\circ} \mathrm{C}$. Ainda, pode ser observado que
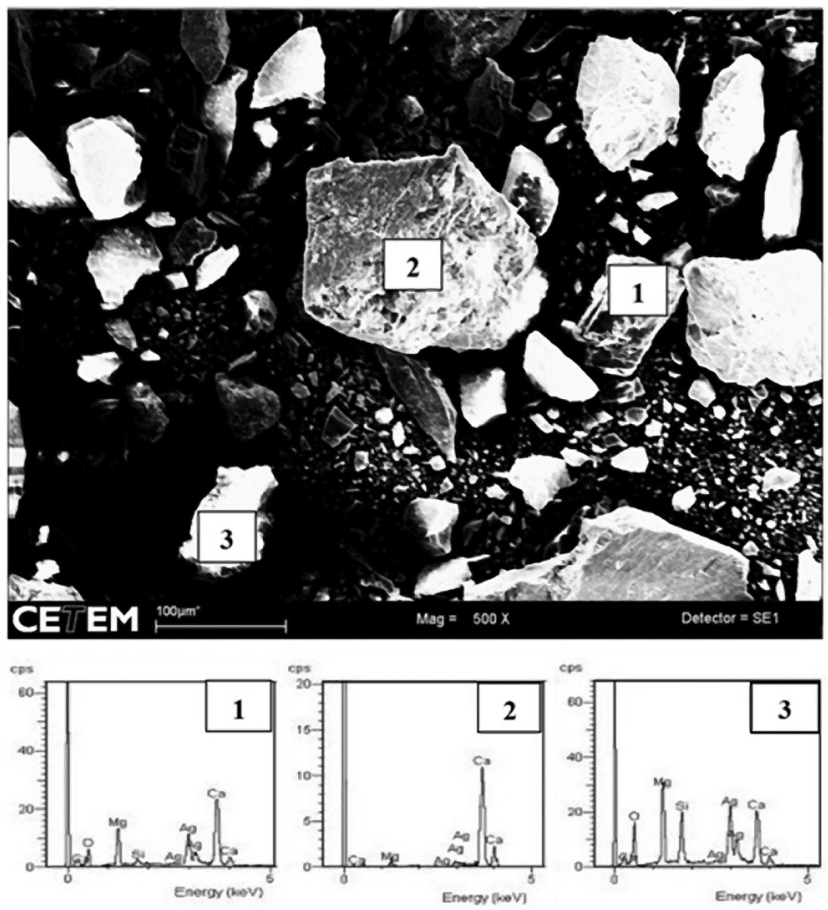

Figura 2. Imagem de MEV do estéril e os constituintes minerais identificados por EDS: (1) dolomita, (2) calcita e (3) diopsídio

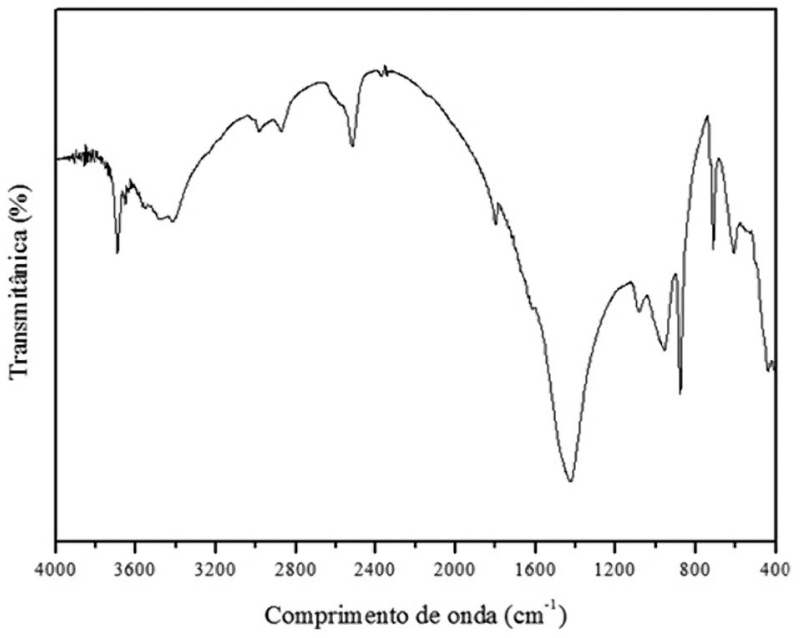

Figura 3. Espectro no infravermelho do estéril na região de $4000-400 \mathrm{~cm}^{-1}$

a perda de massa nessa temperatura é de $30,7 \%$, valor concordante com a perda de massa após calcinação à $1200^{\circ} \mathrm{C}$, da análise química. Contudo, a perda de massa de $2 \%$ observada entre 400 e $600{ }^{\circ} \mathrm{C}$ é atribuída à desidroxilação dos argilos minerais. ${ }^{20} \mathrm{~A}$ perda de massa atribuída ao dióxido de carbono é de $28,7 \%$, ou seja, o estéril possui aproximadamente $40 \%$ de carbonatos.

Conforme a análise dos resultados obtidos na caracterização química e mineralógica do estéril, verifica-se que o mesmo não consta nos anexos A e B da norma NBR-10004, em que são listados os resíduos perigosos. ${ }^{12}$ Ademais, o estéril não possui características de inflamabilidade, corrosividade e reatividade. Na Tabela 2 estão relacionados resultados referentes ao teste de lixiviação do estéril. A análise dos resultados obtidos no extrato lixiviado do estéril revela que as concentrações dos elementos avaliados estão abaixo dos limites definidos no anexo F da norma NBR-10004. Dessa forma, fica descartada a hipótese do estéril ser classificado como resíduo de classe I - perigoso. 


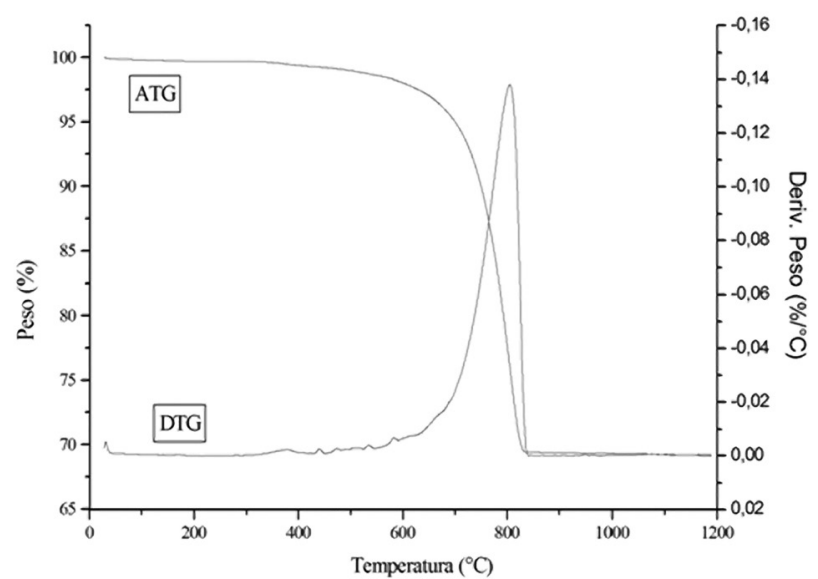

Figura 4. Curva termogravimétrica (ATG) e derivada da curva termogravimétrica $(D T G)$ do estéril em atmosfera oxidante

Tabela 2. Concentração de elementos químicos obtidos no extrato lixiviado do estéril com ácido acético $0,1 \mathrm{~mol} \mathrm{~L}^{-1}$

\begin{tabular}{|c|c|c|c|c|}
\hline $\begin{array}{l}\text { Parâmetros } \\
\text { analisados }\end{array}$ & $\begin{array}{l}\text { Concentração } \\
\text { no lixiviado } \\
\left(\mathrm{mg} \mathrm{L}^{-1}\right)\end{array}$ & $\begin{array}{l}\text { NBR-10004 } \\
\text { limite máximo } \\
\left(\mathrm{mg} \mathrm{L}^{-1}\right)\end{array}$ & $\begin{array}{l}\text { Limite de } \\
\text { detecçã̃o } \\
\left(\mathrm{mg} \mathrm{L}^{-1}\right)\end{array}$ & $\begin{array}{c}\text { Técnicas } \\
\text { analíticas }\end{array}$ \\
\hline Arsênio & ND & 1,0 & 0,03 & ICP-OES \\
\hline Bário & 10,05 & 70,0 & 1,0 & $\mathrm{AA}-\mathrm{N}_{2} \mathrm{O}$ \\
\hline Cádmio & $<0,05$ & 0,5 & 0,05 & AA \\
\hline Chumbo & $<0,2$ & 1,0 & 0,2 & ICP-OES \\
\hline Fluoreto & 2,1 & 150,0 & 0,01 & potenciometria \\
\hline Mercúrio & $<0,001$ & 0,1 & 0,001 & ICP-OES \\
\hline Prata & 0,08 & 5,0 & 0,05 & ICP-OES \\
\hline Selênio & $<1,0$ & 1,0 & 1,0 & ICP-OES \\
\hline $\begin{array}{l}\text { Cromo total } \\
(2 \mathrm{~mm})\end{array}$ & $<0,2$ & 5,0 & 0,2 & $\mathrm{AA}-\mathrm{N}_{2} \mathrm{O}$ \\
\hline $\begin{array}{l}\text { Cromo } \\
\text { hexavalente } \\
(2 \mathrm{~mm})\end{array}$ & $<0,2$ & $\mathrm{NE}$ & 0,2 & Visível \\
\hline $\begin{array}{l}\text { Cromo total } \\
(0,147 \mathrm{~mm})\end{array}$ & $<0,2$ & 5,0 & 0,2 & $\mathrm{AA}-\mathrm{N}_{2} \mathrm{O}$ \\
\hline $\begin{array}{l}\text { Cromo } \\
\text { hexavalente } \\
(0,147 \mathrm{~mm})\end{array}$ & $<0,2$ & NE & 0,2 & Visível \\
\hline
\end{tabular}

ND - não detectável; NE - não especificado.

Os elementos químicos analisados no extrato solubilizado em água após repouso de 7 dias, estão registrados na Tabela 3 e foram comparados com os limites estabelecidos no anexo $\mathrm{G}$ da norma NBR$10004 .^{12}$ A análise dos resultados obtidos no extrato solubilizado mostra que as concentrações dos elementos químicos analisados estão abaixo dos limites definidos na normativa. Portanto, o estéril em estudo, com granulometria abaixo de $2 \mathrm{~mm}$, após ser submetido aos testes de contato dinâmico e estático, à temperatura ambiente, conforme normativas da ABNT, ${ }^{12}$ é classificado como resíduo classe II B - inerte, nas condições estudadas. Esta classificação é decorrente dos resultados obtidos na determinação da concentração dos elementos químicos, tanto no teste de lixiviação como no teste de solubilização, estarem em níveis inferiores aos definidos nos anexos F e G da norma técnica NBR-10004. ${ }^{12}$

Com relação às determinações de cromo total e hexavalente em diferentes granulometrias da rocha, tanto no extrato lixiviado como no extrato solubilizado, verificou-se que mesmo para o estéril com maior área superficial, ou seja, com partículas de granulometria abaixo de $0,147 \mathrm{~mm}$, os teores de cromo total e hexavalente são inferiores
Tabela 3. Concentração de elementos químicos obtidos no extrato solubilizado do estéril

\begin{tabular}{|c|c|c|c|c|}
\hline $\begin{array}{l}\text { Parâmetros } \\
\text { analisados }\end{array}$ & $\begin{array}{c}\text { Concentração } \\
\text { no solubili- } \\
\text { zado }\left(\mathrm{mg} \mathrm{L}^{-1}\right)\end{array}$ & $\begin{array}{l}\text { NBR-10004 } \\
\text { limite máximo } \\
\left(\mathrm{mg} \mathrm{L}^{-1}\right)\end{array}$ & $\begin{array}{l}\text { Limite de } \\
\text { detecção } \\
\left(\mathrm{mg} \mathrm{L}^{-1}\right)\end{array}$ & $\begin{array}{l}\text { Técnicas } \\
\text { analíticas }\end{array}$ \\
\hline Alumínio & ND & 0,2 & 0,04 & ICP-OES \\
\hline Bário & 0,041 & 0,7 & 0,001 & ICP-OES \\
\hline Cádmio & ND & 0,005 & 0,004 & ICP-OES \\
\hline Cianeto & 0,01 & 0,07 & 0,01 & potenciometria \\
\hline Cloreto & 0,95 & 250,0 & 0,01 & CI \\
\hline Cobre & $<0,10$ & 2,0 & 0,1 & AA \\
\hline Ferro & $<0,20$ & 0,3 & 0,20 & AA \\
\hline Fluoreto & 0,20 & 1,5 & 0,01 & $\mathrm{CI}$ \\
\hline Manganês & $<0,05$ & 0,1 & 0,05 & ICP-OES \\
\hline Mercúrio & $<0,001$ & 0,001 & 0,001 & AA \\
\hline Nitrato & 3,3 & 44,3 & 0,01 & $\mathrm{CI}$ \\
\hline Prata & $\mathrm{ND}$ & 0,05 & 0,004 & ICP-OES \\
\hline Sódio & 6,37 & 200,0 & 0,5 & AA \\
\hline Sulfato & 83,2 & 250,0 & 2,0 & $\mathrm{CI}$ \\
\hline Zinco & $<0,10$ & 5,0 & 0,1 & $\mathrm{AA}$ \\
\hline $\begin{array}{l}\text { Cromo total } \\
(2 \mathrm{~mm})\end{array}$ & 0,005 & 0,05 & 0,001 & ICP-OES \\
\hline $\begin{array}{l}\text { Cromo } \\
\text { hexavalente } \\
(2 \mathrm{~mm})\end{array}$ & ND & $\mathrm{NE}$ & 0,017 & Visível \\
\hline $\begin{array}{l}\text { Cromo total } \\
(0,147 \mathrm{~mm})\end{array}$ & 0,004 & 0,05 & 0,003 & ICP-OES \\
\hline $\begin{array}{l}\text { Cromo } \\
\text { hexavalente } \\
(0,147 \mathrm{~mm})\end{array}$ & $\mathrm{ND}$ & $\mathrm{NE}$ & 0,005 & Visível \\
\hline
\end{tabular}

ao limite especificado pela norma técnica. ${ }^{12}$ Além disso, os teores de cromo total e hexavalente no extrato solubilizado das amostras com granulometria de 2 e 0,147 mm são inferiores ao limite de $\mathrm{Cr}(\mathrm{VI})$ aceito pela World Health Organization para água potável, $50 \mu \mathrm{g} \mathrm{L}^{-1} .^{10}$

\section{CONCLUSÕES}

O estéril em estudo é constituído principalmente pelas seguintes fases mineralógicas: dolomita $\left[\mathrm{CaMg}\left(\mathrm{CO}_{3}\right)_{2}\right]$, calcita $\left(\mathrm{CaCO}_{3}\right)$, diopsídio $\left(\mathrm{CaMgSi}_{2} \mathrm{O}_{6}\right)$ e muscovita $\left[\mathrm{KAl}_{2} \mathrm{Si}_{3} \mathrm{AlO}_{10}(\mathrm{OH}, \mathrm{F})_{2}\right]$. Os resultados obtidos pelas técnicas de DRX e MEV/EDS corroboraram os teores obtidos nas análises químicas, que classificaram o estéril como uma rocha ultrabásica. O estudo das fases mineralógicas identificadas no estéril sugere que a rocha tenha sofrido alterações na sua composição mineralógica, visto que foi quase completamente convertida em carbonatos, porém mantém a antigorita $\left[(\mathrm{Mg}, \mathrm{Fe})_{3} \mathrm{Si}_{2} \mathrm{O}_{5}(\mathrm{OH})_{4}\right]$ como seu constituinte mineralógico original. ${ }^{21} \mathrm{~A}$ classificação do estéril quanto ao seu potencial poluidor, na granulometria abaixo de $2 \mathrm{~mm}$, indica que se trata de um resíduo classe II B - inerte, nas condições estudadas, de acordo com a norma técnica NBR-10004. ${ }^{12}$

Diante desses resultados é possível afirmar que o estéril da mina de cromita possui potencial para estudos de aplicação na agricultura, uma vez que não foi classificado como resíduo perigoso e não apresenta quantidades de cromo acima dos limites estabelecidos pela legislação. ${ }^{5,9,11,21}$ As análises revelaram que o estéril possui, na sua composição química, quantidades de cálcio e magnésio adequadas para um corretivo de acidez do solo. ${ }^{4,5}$ Todavia, devido ao estéril não possuir antecedentes de uso agrícola no país, é necessária a realização de testes complementares para avaliar o seu efeito residual e cumulativo em diferentes tipos de solo, com especial atenção para a 
questão do cromo. ${ }^{9,10}$ É aconselhável realizar o acompanhamento da quantidade deste elemento, ao longo do tempo, nos produtos alimentares gerados com a utilização deste estéril como insumo agrícola, tendo como perspectiva a análise de risco, discutida em recente revisão publicada por pesquisadores da EMBRAPA.9,21 Ademais, o estudo deste estéril representa uma contribuição para um melhor aproveitamento da mina de cromita do município de Andorinha/BA, o que minimizaria o impacto ambiental relacionado ao acúmulo desse estéril. Cabe ressaltar que a utilização desse estéril envolve um custo mínimo, uma vez que não será necessário o processamento físico da rocha, a qual se encontra lavrada e prescinde de tratamento químico para a disponibilidade de macronutrientes como cálcio e magnésio.

\section{AGRADECIMENTOS}

Ao CNPq, CAPES e CETEM/MCTI pelo apoio financeiro e infraestrutura para o desenvolvimento deste trabalho. À FERBASA pelo fornecimento das amostras.

\section{REFERÊNCIAS}

1. Fyfe, W. S.; Theodoro, S. H.; Leonardos, O. H.; An. Acad. Bras. Ciênc. 2006, 78, 715; Theodoro, S. H.; Leonardos, O. H.; An. Acad. Bras. Ciênc. 2006, 78, 721; Straaten, P. V.; An. Acad. Bras. Ciênc. 2006, 78 , 731.

2. Mello Filho, C. H. R.; Oliveira, J. R.; Espinosa, D. C. R.; Tenório, J. A. S.; Rev. Esc. Minas 2010, 63, 271; Corrêa, J. C.; Bull, L. T.; Crusciol, C. A. C.; Moraes, M. E.; Rev. Bras. Ciênc. Solo 2009, 33, 263; Medeiros, J. C.; Albuquerque, J. A.; Mafra, A. L.; Batistella, F.; Grah, J.; Rev. Bras. Ciênc. Solo 2009, 33, 1657; Corrêa, J. C.; Bull, L. T.; Crusciol, C. A. C.; Marcelino, R.; Mauad, M.; Pesq. Agropec. Bras. 2007, 42, 1307; Ribeiro, F. R.; Filho, F. B. E.; Fabris, J. D.; Mussel, W. N.; Novais, R. F.; Rev. Bras. Ciênc. Solo 2007, 31, 939; Silverol, A. C.; Filho, L. M.; Rev. Bras. Agroecologia 2007, 2, 703; Prado, R. M.; Natale, W.; Fernandes, F. M.; Corrêa, M. C. M. R.; Rev. Bras. Ciêc. Solo 2004, 28, 197; Prado, R. M.; Fernandes, F. M.; Sci. Agric. 2000, 57, 739.

3. Merwe, W. V. D.; Environ. Sci. Technol. 2010, 44, 1806; RodriguezJorda, M. P.; Garrido, F.; Garcia-Gonzalez, M. T.; J. Hazard. Mater. 2010, 175, 762; Szumera, M.; Wacławska, I.; Olejniczak, Z.; J. Therm. Anal. Calorim. 2010, 99, 879; Torkashvand, M. A; Afr. J. Agric. Res. 2010, 5, 3082; Jayasinghe, G. Y.; Tokashiki, Y.; Kitou, M.; Water, Air, Soil Pollut. 2009, 204, 29; Wacławska, I.; Szumera, M.; J. Alloys Compd. 2009, 468, 246.

4. Teixeira, A. M. S.; Sampaio, J. A.; Garrido, F. M. S.; Medeiros, M. E.; Bertolino, L. C.; Pérez, D. V. Em Anais do II Simpósio de Minerais Industriais do Nordeste; Luz, A. B.; Baltar, C. A. M.; Bertolino, L. C., eds.; CETEM: Rio de Janeiro, 2010, cap. 3.

5. Brasil, Ministério da Agricultura, Pecuária e Abastecimento; Instrução Normativa $n^{\circ} 28$, de 27/07/2007, Diário Oficial da União, 31/07/2007; Brasil, Ministério da Agricultura, Pecuária e Abastecimento; Instrução Normativa $n^{\circ} 35$, de 04/07/2006, Diário Oficial da União, 12/07/2006; Brasil, Ministério da Agricultura, Pecuária e Abastecimento; Instrução Normativa $n^{\circ}$ 27, de 05/06/2006, Diário Oficial da União, 09/06/2006.

6. Boschetti, T.; Toscani, L.; Chem. Geo. 2008, 257, 76; Pereira, D.; Yenes, M.; Blanco, J. A.; Peinado, M.; Geo. Soc. 2007, 271, 55; DNPM; Anuário Mineral Brasileiro, $35^{a}$ ed., DNPM: Brasília, 2006; Yalçin, H.; Bozkaya, O.; Clays Clay Miner. 2006, 54, 333.

7. Luz, A. B.; Lins, F. A. F. Em Tratamento de Minérios; Luz, A. B.; Sampaio, J. A.; França, S. C. A., eds.; CETEM: Rio de Janeiro, 2010, cap. 1; Luz, A. B.; Lins, F. F.; Rochas e Minerais Industriais; $2^{\mathrm{a}}$ ed., CETEM: Rio de Janeiro, 2005.
8. Manhães, J. P. V. T.; de Holanda, J. N. F.; Quim. Nova 2008, 31, 1301; Oliveira, M. R. C.; Martins, J.; Quim. Nova 2003, 26, 5.

9. Andrade, J. C.; Abreu, M. F.; Análise Química de Resíduos Sólidos para Monitoramento e Estudos Agroambientais, Instituto Agronômico: Campinas, 2006.

10. Soares, R.; Carneiroa, M. C.; Monteiroa, M. I. C.; Henrique Júnior, S. S.; Pontesa, F. V. M.; Silva, L. I. D.; Alcover Neto, A.; Santelli, R. E.; Chem. Speciation Bioavailability 2009, 21, 153; Matos, W. O.; Nóbrega, J. A.; Souza, G. B.; Nogueira, A. R. A.; Quim. Nova 2008, 31, 1450; Mandiwana, K. L.; Panichev, N.; Kataeva, M.; Siebert, S.; J. Hazard. Mater. 2007, 147, 540; Oze, C.; Bird, K. D.; Fendorf, S.; Proc. Natl. Acad. Sci. U. S. A. 2007, 104, 6544; Surita, C. A.; Saito, M. M.; Ellert, N.; R. Bras. Recursos Hídricos 2003, 8, 47; Kumar, S. J.; Ostapczuk, P.; Emons, H.; J. Anal. Chem. 1997, 359, 171.

11. Brasil, Ministério do Meio Ambiente; Resolução $n^{o} 420$, de 28/12/2009, Diário Oficial da União, 30/12/2009.

12. ABNT; Resíduos Sólidos - Classificação, ABNT: Rio de Janeiro, 2004 (norma técnica NBR-10004); ABNT; Procedimento para obtenção de extrato lixiviado de resíduos sólidos, ABNT: Rio de Janeiro, 2004 (norma técnica NBR-10005); ABNT; Procedimento para obtenção de extrato solubilizado de resíduos sólidos, ABNT: Rio de Janeiro, 2004 (norma técnica NBR-10006); ABNT; Amostragem de resíduos sólidos, ABNT: Rio de Janeiro, 2004 (norma técnica NBR-10007).

13. Oliveira, M. L. M.; Aquino, J. A. Em Tratamento de Minérios: Práticas Laboratoriais; Sampaio, J. A.; França, S. C. A.; Braga, P. F. A., eds.; CETEM/: Rio de Janeiro, 2007, cap. 1.

14. ICDD; International Center for Diffraction Data; PDF02 dataset, 2006.

15. Sah, S. L.; Encyclopaedia of Petroleum Science \& Engineering: Petrografy: Chemical and Quasi-chemical classification, Kalpaz Publication: Delhi, 2005; Potts, P. J.; A Handbook of Silicate Rock Analysis, Chapman \& Hall: New York, 1987.

16. Korndörfer, G. F.; Pereira, H. S.; Camargo, M. S.; Silicatos de cálcio e Magnésio na Agricultura, $2^{\mathrm{a}}$ ed., Grupo de Pesquisa "Silício na Agricultura": Uberlândia, 2003; Fadigas, F. S.; Sobrinho, N. M. B. A.; Mazur, N.; Anjos, L. H. C.; Freixo, A. A.; R. Bras. Eng. Agric. Ambiental 2006, 10, 699.

17. Franco, D. V.; Rodrigues, M. L. K.; Bavaresco, J.; Jardim, W. F.; Quim. Nova 2011, 34, 1255.

18. Changwen, D.; Linker, R.; Shaviv, A.; Appl. Spectrosc. 2007, 61, 1063 ; Nyquist, R. A.; Kagel, R. O.; Infrared Spectra of Inorganic Compounds, Academic Press Inc.: New York, 1997; Nakamoto, K.; Infrared Spectra of Inorganic and Coordination Compound; John Wiley \& Sons: New York, 1970; La Russa, M. F.; Ruffolo, S. A.; Barone, G.; Crisci, G. M.; Mazzoleni, P.; Pezzino, A.; Int. J. Spectroc. 2009, 2009, 1.

19. Böke, K.; Çizer, Ö.; Ipekoğlu, B.; Uğurlu, E.; Şerifaki, K.; Toprak, G.; Construction and Building Materials 2008, 22, 866.

20. Teixeira, A. M. S.; Garrido, F. M. S.; Sampaio, J. A.; Medeiros, M. E. Em XXIII Encontro Nacional de Tratamento de Minérios e Metalurgia Extrativa; Brum, I. A. S.; Sampaio, C. H., orgs., Porto Alegre 2009, vol. 2, cap. 10.; Teixeira, A. M. S.; Sampaio, J. A.; Garrido, F. M. S.; Medeiros, M. E.; The Minerals, Metals \& Materials Society - Extraction \& Processing 2010, 1, 293.

21. Marchi, G.; Guilherme, L. R. G.; Silva, C. A.; Gonçalves, V. C.; Elementos-traço e sua relação com a qualidade e inocuidade de fertilizantes, corretivos agrícolas e resíduos orgânicos no Brasil, EMBRAPA Cerrados: Planaltina, 2009. 